\title{
Characterization of Hepatocellular Carcinoma (HCC) in CT Images using Texture Analysis Technique
}

\author{
Abdoelrahman Hassan A. B. ${ }^{1}$, Samia A. F. Ahmed ${ }^{2}$, MEM Gar-elnabi ${ }^{3}$, M. A. Ali Omer ${ }^{5}$, \\ Asma I. Ahmed ${ }^{4}$ \\ ${ }^{1,3,4}$ Collage of Medical Radiologic Science, Sudan University of Science and Technology, Khartoum, Sudan \\ ${ }^{2}$ Radiological sciences Department, College of Applied medical sciences, Najran University, Najran, KSA \\ ${ }^{5}$ Radiologic Technology Department, Collage of Applied Medical Science, Qassim University, Buraiah, KSA
}

\begin{abstract}
A new approach to texture characterization from dynamic CT scans of the liver is presented. This study was aimed to use the texture analysis and classification methods to characterize the hepatocellular carcinoma (HCC), liver and other abdominal regions in CT images using image processing program (IDL, interactive data language). Tri-phasic Multi detectors CT with contrast enhanced images of 200 adult subjects with hepatocellular carcinoma were used as entry data. Tiff format was created as IDL variables and then using 3x3 window the image was scanned and based on the image histogram the selected feature also called FOS was calculated using this window. Linear discriminant analysis was used for the tissue classification. The study found that the HCC texture reveal a different underlying pattern compared to the liver and other abdominal tissues with classification sensitivity and specificity 96.5\% and 86.6\% respectively, and the combination of the texture features throughout the different tri-phasic image phases provide the highest predictive overall accuracy of $89.1 \%$ using linear discriminant analysis.
\end{abstract}

Keywords: FOS, HCC, CT images, classification.

\section{Introduction}

Hepatocellular carcinoma (HCC) is the most frequent malignant liver tumor, which it can be primary or secondary tumors. Computerized methods, which extract information from medical images, are being widely used studies nowadays in order to replace the old methods through a new, non-invasive technology. Texture is a fundamental visual property which is essential especially in tissue characterization and in pathological structures recognition. Texture-based methods are implemented in association with classifiers in order to perform automatic tumor differentiation for various kinds of organs $[1,2$, and 3$]$.

Computed Tomography (CT) in now a widely applied tool for diagnosis of hepatic tumors. The visual analysis of image series, acquired usually before a contrast injection and during its propagation, enables doctors to detect lesions and to recognize a certain extent, the type of pathology. However, in most cases, visual inspection of CT scans could not be sufficient for proper image interpretation. Even for experienced radiologists, the correct differentiation of tumor affected tissue is a difficult task. The definitive diagnosis often requires invasive procedures like needle biopsy or even surgery, which carry a risk of complications. New computeraided image processing methods (in particular methods of their texture analysis), in combination with effective classification algorithms, can considerably improve the accuracy of the diagnosis. Extracting the information not normally detected by the human eye, those techniques could reduce or even eliminate the necessity of performing the invasive techniques [4].
An objective and clear characterization of image regions is one of the crucial problems to deal with when a computer aided image analysis is performed. One of the most useful sources of information about analyzed image regions could be their texture [5]. The texture analysis consists in extracting a set of numerical parameters (so-called texture features) to characterize Regions of Interest (ROIs) defined in the organs under study. Each of the texture parameters expresses a specified property of the texture, like coarseness, homogeneity, or the local contrast. So far, a great variety of texture features extraction methods has been investigated [6]. The Liver CAD System consists of Preprocessing, Segmentation of Liver, ROI Analysis and Classification. Preprocessing is to decrease of artifacts, image noise reduction and leveling of image quality. Segmentation means extracting the liver from abdominal CT and also extracting tumor according to its intensity profile. Classification applies any classifier to identify the liver disease based on textural features. In radiology computer-aided diagnosis (CAD) are procedures in medicine that help doctors in the analysis of medical images. CAD system can only provide a second opinion and cannot replace radiologist's reposts. [7]

A lot of liver texture analysis techniques have been proposed in the past for CT imaging modalities to extract useful features for reliable liver tissue classification. The efforts have been put in surveying the efficiency of textural analysis techniques devised for all imaging modalities [8].

Texture analysis is an essential issue in image processing. It comprises a set of mathematical techniques used to quantify the different gray levels within an image in terms of intensity and distribution. Texture represents the spatial arrangement 


\section{International Journal of Science and Research (IJSR) \\ ISSN (Online): 2319-7064}

Index Copernicus Value (2013): 6.14 | Impact Factor (2014): 5.611

of pixels' gray levels in a region. So, it can be divided into two classes: periodic texture and random texture. Consequently, we can distinguish the structural approaches and the statistic approaches to calculate a number of mathematical parameters that characterize the texture. Structural approaches are more suited to the study of periodic or regular textures. However, statistic approaches are used to characterize fine and non-homogeneous structures without apparent regularity. That is why; this type of method is generally applied in medical imaging [7]. A statistical approach perceives a texture as a quantitative measure of the arrangement of intensities in an area. Statistical methods can be categorized into first order, second-order and higher-order and spectral statistics, based on the number of pixels used to define the feature. $[10]$

First order statistics measures (FOS) based on the image histogram to calculate texture. The main advantage of this approach is its simplicity through the use of standard descriptors (e.g. mean and variance) to characterize the data. For any surface, or image, grey-levels are in the range $0 \leq \mathrm{i} \leq$ $\mathrm{Ng}-1$, where $\mathrm{Ng}$ is the total number of distinct grey-levels. If $N$ (i) is the number of pixels with intensity $i$ and $M$ is the total number of pixels in an image, it follows that the histogram, or pixel occurrence probability, is given by,

$$
P(i)=\frac{N(i)}{M} .
$$

The main image processing discipline in which texture analysis techniques are used are classification, segmentation and synthesis. In image classification the goal is to classify different images or image regions into distinct groups [12]. Texture analysis methods are well suited to this because they provide unique information on the texture, or spatial variation of pixels, of the region where they are applied. In image segmentation problems the aim is to establish boundaries between different image regions. Synthesizing image texture is important in three-dimensional (3D) computer graphics applications where the goal is to generate highly complex and realistic looking surfaces. [13].

In general seven features commonly used to describe the properties of the image histogram, and therefore image texture, are computed. These are: mean; variance; coarseness; skewness; kurtosis; energy; and entropy. [11]

\section{Material and Method}

A database of 600 images (200 images for each acquisition phase) from 200 patients was gathered. The acquisitions were performed with MDCT device and the standardized acquisition protocol was applied: helical scanning, with slice thickness $5 \mathrm{~mm}$. For each patient, an appropriate amount of the contrast material was chosen (about $100 \mathrm{ml}$ ), and the injection was performed at $3 \mathrm{ml} / \mathrm{s}$. The acquisition of the images were acquired firstly pre-contrast administration, then in the arterial phase about 20 seconds after the contrast product injection. Images corresponding to the portal phase were acquired with delay of 50-60 s. All images had a size of $512 \times 512$ pixels with 8 -bit gray levels and were represented in DICOM format. 6 features from FOS (six of each acquisition phase) which are mean; variance; skewness; kurtosis; energy; and entropy are computed from the intensity function of the image and were calculated for each ROI through all phases .

\section{Result Presentation}

Table 1: Showed the classification accuracy of the HCC using linear discriminant analysis:

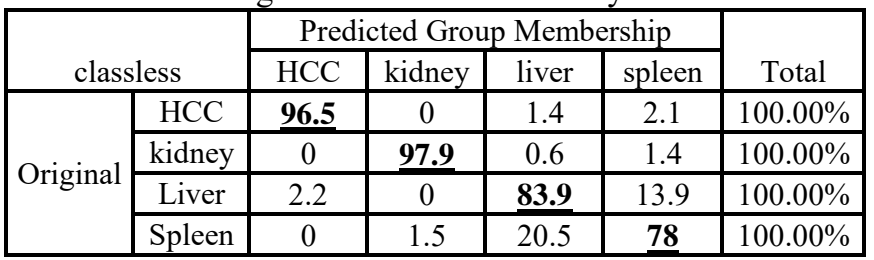

$89.1 \%$ of original grouped cases correctly classified.

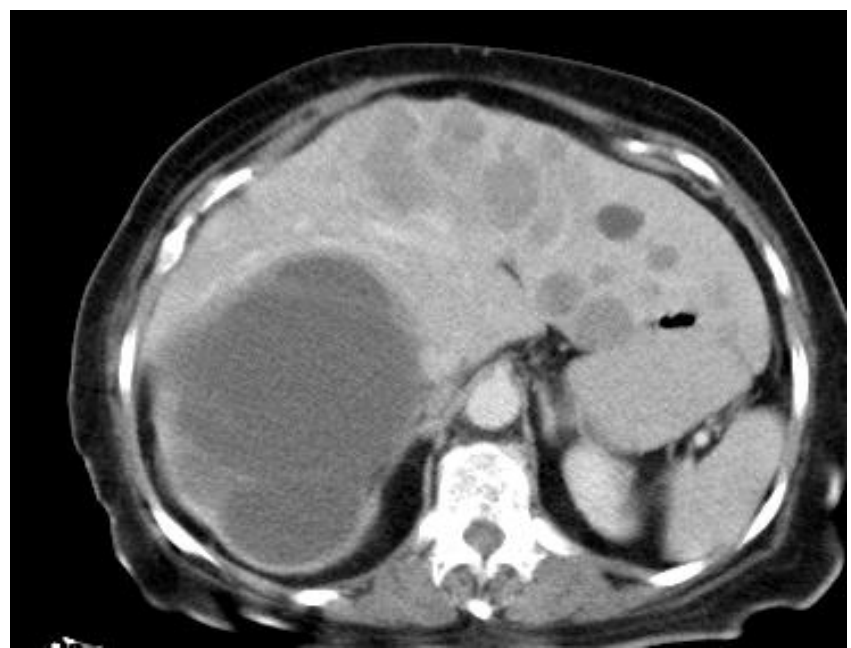

Figure 1: The original image CT- with contrast demonstrate the liver lesion.

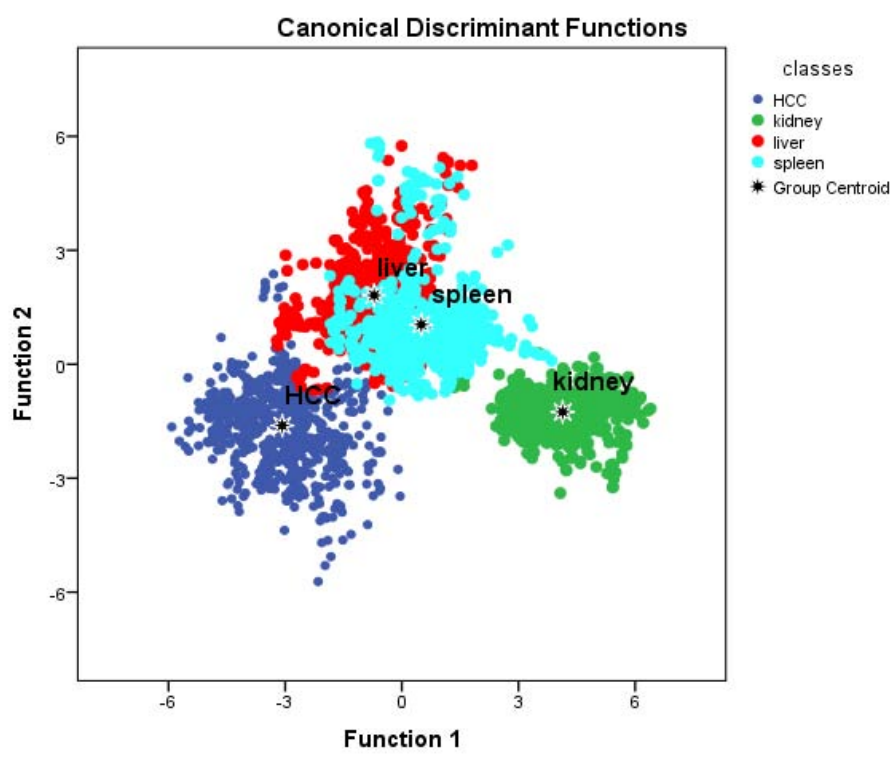

Figure 2: Scatter plot generated using discriminate analysis function for four classes represents: HCC, Liver, Kidney and Spleen. 


\section{International Journal of Science and Research (IJSR) \\ ISSN (Online): 2319-7064}

Index Copernicus Value (2013): 6.14 | Impact Factor (2014): 5.611

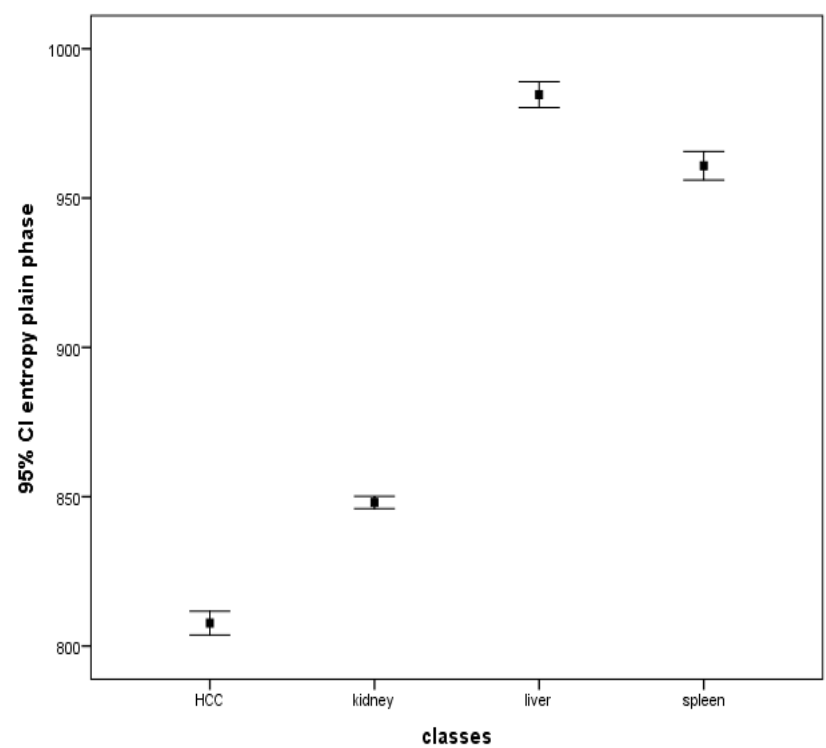

Figure 3: Classification based on entropy for plain CT series

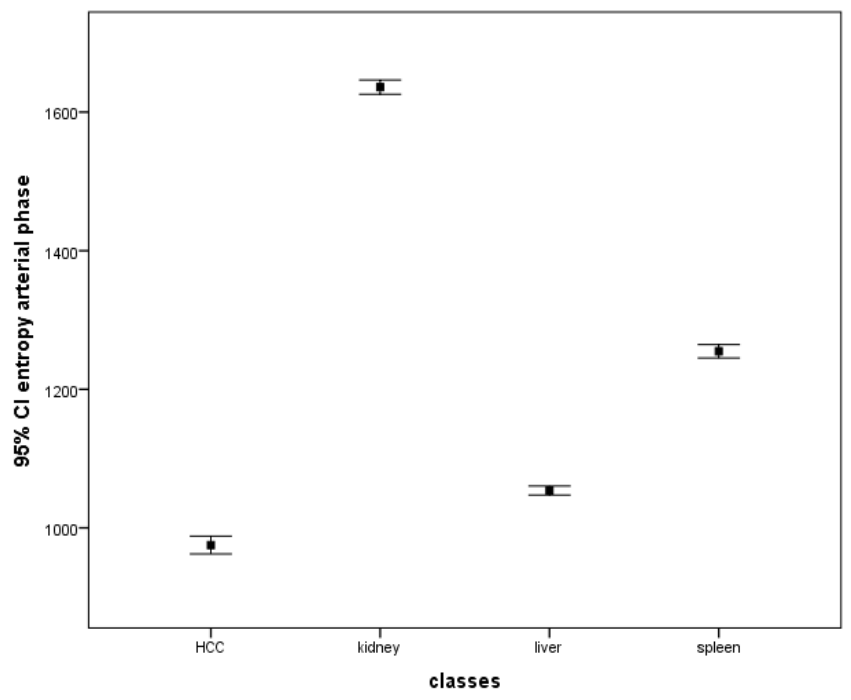

Figure 4: Classification based on entropy for arterial phase CT series

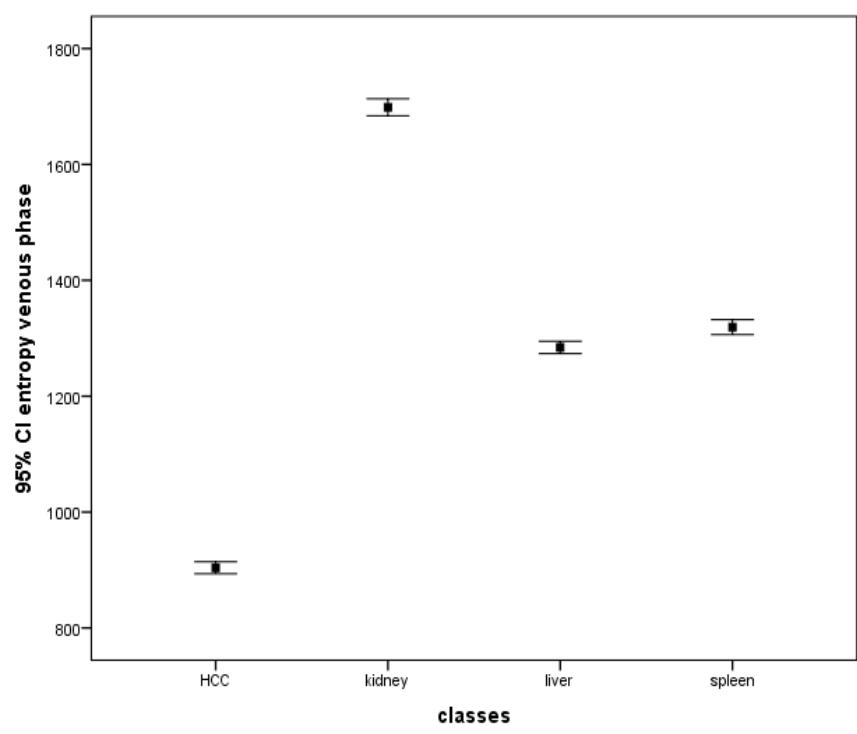

Figure 5: Classification based on entropy for Porto venous phase CT series

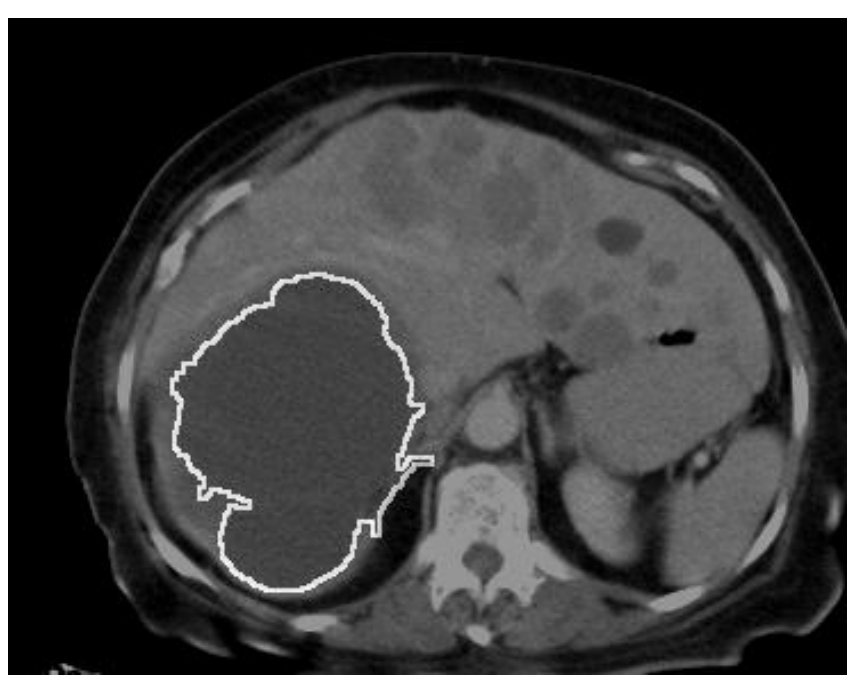

Figure 6: Showed the lesion (HCC) drawn using intensity profile of the CT image in which the tumor borders clearly outlined.

\section{Discussion}

Firstly the classification was aimed to extract these feature from the image based on the normal image histogram where the primary image (figure 1.) was converted into tiff format as an input image for IDL image processing program, then a window of $3 \times 3$ was created in order to scan the image then the feature were extracted for three different phases of CT series; mean; variance; coarseness; skewness; kurtosis; energy; and entropy. All these feature were calculated for all images and then the data were ready for discrimination which was performed using step-wise technique in order to select the most significant feature that can be used to classify the HCC in tri-phasic CT imaging (arterial, Porto-venous and plain (without contrast images)) and the result showed that:

Table (1). Linear discreaminant function and the classification accuracy of each class was presented in which $96.5 \%$ of HCC was correctly classified and $97.9 \%, 83.9 \%$ and $78.0 \%$ classification sensitivity for kidney, rest of liver tissue and spleen respectively with classification specificity of $86.6 \%$ were computed from gray level histogram and the results are represented that there is a well concentration of features around the class centers which give a remarkable difference among the four classes especially between the $\mathrm{HCC}$ and normal liver tissues in the entropy in plain, arterial and venous phases (Fig 1, 2, 3, 4, and 5) however, No clear difference in the entropy was seen between the HCC and liver in the arterial phase (Fig 4.). Among all image phases, HCC reveal different value which, was low and relatively static (low entropy variation).

Linear discriminate analysis were used to classify the HCC, and normal abdominal tissues, so the features of the classified regions of the whole images (as raw data) were classified furthers. The result of the classification showed that the HCC were classified well from the normal liver tissues even though it has characteristics similar to surrounding tissue, and the texture reveal a different underlying pattern compared to the liver and other abdominal tissues with classification sensitivity $96.5 \%$, and the combination of the texture features throughout the different 


\section{International Journal of Science and Research (IJSR) \\ ISSN (Online): 2319-7064}

Index Copernicus Value (2013): 6.14 | Impact Factor (2014): 5.611

tri-phasic image phases provides the highest predictive overall accuracy of $89.1 \%$ using linear discriminant analysis Same result achieved by D. Duda 2006 which presented that (Characterization of the texture from multiphase CT images was presented. Images with the same slice location and corresponding to three typical acquisition moments were analyzed simultaneously. In this way the texture evolution during the propagation of the contrast product was taken into account. The method was applied to recognizing normal liver and its two main primary tumors. Experiments with various sets of texture parameters and two classification methods showed that a simultaneous analysis of texture parameters derived from three subsequent acquisition moments considerably improved the classification accuracy) [9].

Also the lesion was examined and the image was tested using $3 \times 3$ matrix size (window) based on the intensity profile of the scanned image and this performed in order to outline the tumor accurately and this can be used for planning process of radiotherapy treatment.

Finally, excellent discrimination between hepatocellular carcinoma and other abdominal organ can be established on the basis as few as three optimal feature among the 18 texture characteristics tested. This serves as a second method to perform more characterization of the tumor.

\section{Conclusion}

This study conclude that HCC and other abdominal organ in CT images for simplicity can be diagnosed and classify by using the following simple equation after extracting the associated features using a window of $3 \times 3$ pixel from the region of interest; the biggest classification score assume the tissue type :

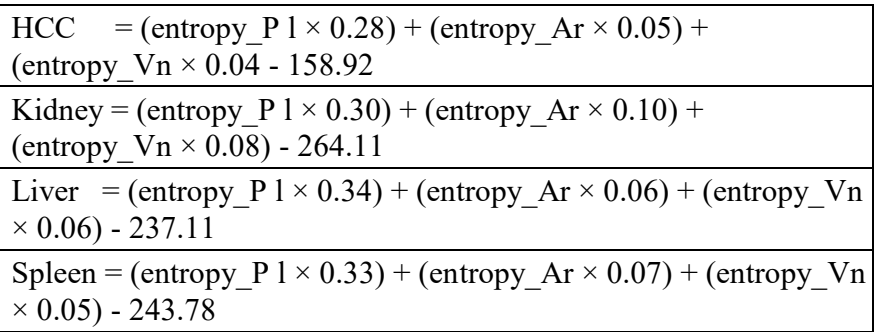

\section{References}

[1] Chikui, T., K. Tokumori, K. Yoshiura, K. Oobu, S.Nakamura, K. Nakamura (2005), Sonographic characterization of salivary gland tumors by fractal analysis , Ultrasound in Medicine and Biology, Vol. 31, No. 10, pp. 1297-1304

[2] Yoshida, H.,D.Casalino, B. Keserci, A. Coskun, O. Ozturk and A. Savranlar (2003), Wavelet packet- based texture analysis for differentiation between benign and malignant liver tumors in ultrasound images, Physics in Medicine and Biology, No. 48, pp. 3735-375

[3] Madabhushi, A., M.D. Felman, D.N. Metaxas, J. Tomaszeweski, D. Chte (2005), Automated Detection of Prostatic Adenocarcinoma From High-Resolution Ex
Vivo MRI, IEEE .Transactions on Medical Imaging, December 2005, pp. 1611-1626

[4] Bruno A., Collorec R., Bezy-Wendling J., Reuze P., Rolland Y.: Texture analysis in medical imaging, In: Roux C., Coatrieux J. L. (Eds.): Contemporary Perspectives in Three-dimensional Biomedical Imaging, IOS Press 1997, 133-164.

[5] Haralick R. M.: Statistical and structural approaches to texture, Proc. IEEE 1979, 67, 786-804.

[6] Galloway M. M.: Texture analysis using gray level run lengths. Computer Graphics and Image Processing 1975, 4, 172-179.

[7] Olfa Ben Sassi, Lamia sellami1, Mohamed Ben Slima1, improved spatial gray level dependence matrices for texture analysis, International Journal of Computer Science \& Information Technology (IJCSIT) Vol 4, No 6, December 2012, pp 209- 219

[8] Rathore S, Iftikhar MA, Hussain M, Jalil A (2011) Texture analysis for liver segmentation and classification: a survey. In: Proc. of International Conference of IEEE on Frontiers of Information Technology, pp 121-126.

[9] D. Duda, M. Krętowski, J. Bezy-Wendling, Texture Characterization for Hepatic Tumor Recognition in Multiphase CT , Biocybernetics and Biomedical Engineering, Volume 26, Number 4,2006, pp. 15-24

[10] Gunasundari S, Janakiraman A Study of Textural Analysis Methods for the Diagnosis of Liver Diseases from Abdominal Computed Tomography ,International Journal of Computer Applications (0975 - 8887) Volume 74- No.11, pp 7-13 July 2013

[11] Tuceryan, M. \& Jain, A.K. (1998). Texture analysis. In: Chen, C.H; Pau, L.F. \& Wang, P.S.P., (eds). The handbook of pattern recognition and computer vision. 2nd ed. World Scientific Publishing Co., ISBN 9-81023071-0, Singapore.

[12]Pietikainen, M.K. (ed) (2000). Texture analysis in machine vision, World Scientific Publishing, 981-024373-1, Singapore.

[13] Mirmehdi, M.; Xie, X. \& Suri, J. (eds) (2008) Handbook of texture analysis, Imperial College Press, 184816-115-8, UK.

[14] Kumar SS, Moni RS, Rajeesh J (2012) Liver tumor diagnosis by gray level and contourlet coefficients texture analysis. International Conference on Computing, Electronics and Electrical Technologies ,pp 557 - 562

[15]E-Liang Chen, Pau-CHoo Chung, Ching-Liang Chen, Hong-Ming Tsai and Chein I Chang, "An Automatic Diagnostic system for CT Liver Image Classification", IEEE Transactions Biomedical Engineering, vol 45, no. 6, pp. 783-794, June 1998.

[16] Yu-Len Huang, Jeon-Hor Chen, Wu-Chung Shen "Computer-Aided Diagnosis of Liver Tumors in Nonenhanced CT Images" Department of Computer Science and Information Engineering, Tunghai University, Mid Taiwan, Journal of Medical Physics, Vol. 9, pp. 141150, 2004.

[17] S. Gr. Mougiakakou1, I. Valavanis1, K. S. Nikita1, A. Nikita2, D. Kelekis2 Characterization of CT Liver Lesions Based on Texture Features and a Multiple Neural Network Classification Scheme, 2003 IEEE, pp.1287- 1291 .

\section{Volume 5 Issue 1 January 2016}




\section{Author Profile}

Mr. Abdoelrahman Hassan Ali Bakry (Sudan) received the (B.Sc.) and (M.Sc.) in radiotherapy technology from College of Medical radiological Science, Sudan University of Science and Technology in 2013 and 2015 respectively. During 2013 up to date, he is staying in College of Medical radiological Science, Sudan University of Science and Technology, Radiology Department, Antalya Medical Center and Elnileen Diagnostic Medical Center; also he has been active in Computerized Texture Analysis, Radiotherapy-Oncology, and diagnostic radiology, Medical physics, ultrasound and Nuclear Medicine researches. Now he is assistant teacher at SUST also (2014).

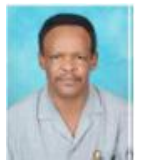

Assoc. proff. Dr. Mohamed Elfadil Mohamed Garelnabi (Sudan) awarded the B. Sc. in Radiotherapy and Nuclear Medicine (1987) and M.Sc. in Radiation Therapy (2000-SUST) and Ph. D. degree in Medical Physics (Natal University-South Africa) in 2007. During 1996-2012 he has been working as lecturer as well as Associate Prof. at SUST department of Radiation therapy. Also he has been active in Computerized Texture Analysis, RadiotherapyOncology, Ultrasound and Nuclear Medicine researches.

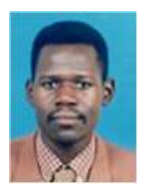

Assoc. proff. Dr. Mohammed A. Ali Omer (Sudan) awarded the B. Sc. in Radiotherapy and Nuclear Medicine (1995) and M.Sc. in Medical Physics (2002SUST) and Ph. D. degree in Medical Physics (UPM) in 2007. During 1996-2012 he has been working as lecturer as well as Associate Prof. recently at SUST-department of Radiation therapy. Now he works in Qassim University in Kingdom of Saudi Arabia. Also has been active in polymer processing Nanocomposites, Bioengineering and cancer researches. 